\title{
GENERANDO OPINIÓN CIUDADANA EN LA CULTU- RA DIGITAL A TRAVÉS DE LOS MAPAS ${ }^{1}$
}

\author{
María ZÚÑIGA-ANTÓN, María SEBASTIÁN LÓPEZ, Ángel PUEYO \\ CAMPOS, Raúl POSTIGO VIDAL, Celia SALINAS SOLE, Aldo ARRANZ \\ LÓPEZ \\ Grupo de Estudios en Ordenación del Territorio (GEOT), Departamento de \\ Geografía y Ordenación del Territorio, Instituto de Investigación en Ciencias \\ Ambientales (IUCA,)Universidad de Zaragoza (mz@unizar.es)
}

Recibido: 20/06/2015

Aceptado: 03/09/2015

RESUMEN: El contexto actual de cambio y transformación socioespacial experimentado en los últimos años incrementa la necesidad de un conocimiento exhaustivo y actualizado de los espacios de vida. A su vez, se pone de manifiesto la exigencia ciudadana de tener a su alcance herramientas que le permitan el seguimiento de las actuaciones públicas que afectan a su territorio. Conscientes de esta situación, los investigadores están en la posición de proporcionar alternativas eficientes, como la cartografía temática, que garanticen la interacción entre el ciudadano, la información geográfica y el espacio.

Una exposición documental contextualizada, es una propuesta que ha demostrado ser eficiente ya que facilita la creación de opinión sobre las actuaciones y permite al ciudadano realizar un seguimiento y jerarquización de las mismas. Ejemplo de esta estrategia es la exposición Zaragoza Mapa a Mapa que se diseñó como un proyecto cartográfico a través de Sistemas de Información Geográfica, que incluía diferentes niveles de complejidad para permitir el entrenamiento de los visitantes en la lectura de los mapas. Parte importante del éxito de la exposición radicó en la combinación del espacio digital y físico, a través de pizarras interactivas, cartografía animada y el uso de redes sociales como punto de encuentro entre el ciudadano, su espacio y las propuestas de actuación en su entorno.

\footnotetext{
${ }^{1}$ Este trabajo es el resultado de los convenios de colaboración entre el Grupo de Estudios en Ordenación del Territorio (GEOT) de la Universidad de Zaragoza y el Ayuntamiento de Zaragoza, Ebropolis y el Instituto Geográfico Nacional. Las actualizaciones del mismo se enmarcan en el proyecto «Herramientas cartográficas para una gobernanza inteligente en las ciudades digitales: análisis territorial de las condiciones de vida» (CSO2013- 46863-C3-3-R) del Programa Estatal de Investigación, Desarrollo e Innovación Orientada a los Retos de la Sociedad del Ministerio de Economía y Competitividad de España y en los contratos de investigación con la Universidad de Zaragoza 2013/1126 «Organización de la exposición Zaragoza Mapa a Mapa», y 2014/0466 «Referenciación de la información del padrón municipal por portales y desarrollo de herramientas cartográficas para una gobernanza inteligente en la ciudad de Zaragoza».
} 
PALABRAS CLAVE: Neogeografía, exposición, cartografía temática, demografía, ciudad, sociedad, redes sociales.

MAPS AS A TOOL FOR CITIZEN OPINION IN THE DIGITAL CULTURE

ABSTRACT: In the present context of change and sociospatial transformation of cities the need of an exhaustive and updated knowledge about living spaces has increased. At the same time, citizens call for tools to follow the effectiveness of public actuations. Aware of this situation, researchers can provide cartographic instruments to ensure the people-geoinformation interaction.

A documentary contextualized exposition is an efficient proposal to develop opinion and to track and prioritize public investments. An example of this strategy was the exposition called «Zaragoza map to map» designed as a multilevel project to train visitors in map reading using Geographic Information Systems. The success of the exposition was mainly associated to the combination of digital and real spaces by using interactive panels, animated cartography and social networks as a meeting point between the citizens, the space and their management proposals.

KEY WORDS: Neogeography, exposition, thematic map, demography, city, society, social networking.

\section{INTRODUCCIÓN}

El concepto de Neogeografía conlleva la reinvención de la ciencia geográfica puesto que supone la utilización por parte de usuarios no expertos de técnicas y herramientas propias de esta ciencia para uso personal y comunitario (GOODCHILD, 2008: 1).

Este tipo de usuarios han encontrado en las herramientas geográficas una ventana al mundo que conocen, y respecto al cual pueden interpretar y generar nueva información para favorecer los procesos de participación, gobernanza y democracia colaborativa. La proximidad y familiaridad con el objeto de una ciencia es el factor más importante que condiciona el alcance que la misma puede adquirir en la sociedad (GOODCHILD, 2008: 2). Ciertamente todo el mundo controla su propio entorno, todo el mundo es experto en geografía en la medida en la que ésta es su escenario de vida y necesita conocerlo para desenvolverse diariamente. Entendiendo que la Neogeografía desdibuja los límites entre los roles tradicionales de productor, comercializador y consumidor de información geográfica (RANA et al, 2007: 5), se puede comprender que, en el contexto de cambio de paradigma socioespacial que viven las ciudades (CALvo et al, 2011:5), se ponga de manifiesto la necesidad de un conocimiento aún más exhaustivo y actualizado del espacio de vida por parte de usuarios no expertos.

Esto enlaza con el concepto de «ciencia ciudadana», entendida como la participación de personas voluntarias en una investigación (DICKINSON et al, 2010: 1) actividad que tiene el potencial de ampliar el alcance de las investigaciones y mejorar la capacidad de recopilar datos científicos (COHN, 2008: 
1). Personas con un grado de implicación social elevado pueden contribuir con puntos de vista e información valiosa sobre su entorno, es lo que se denomina «Información Geográfica Colaborativa» (VGI en sus siglas en inglés) (ELWOOD, 2008: 133).

Por otro lado debe comprenderse que los profesionales de esta ciencia manejan una serie de competencias como (i) razonar más allá de la observación desarrollando nuevas teorías y generalizaciones, (ii) comprobar la utilidad de una teoría comparando la predicción con la observación de un espacio o (iii) utilizar herramientas sofisticadas para conseguir análisis más profundos (GOODCHILD, 2008: 12). Es por esto que los especialistas encuentran en herramientas colaborativas, como las cartográficas un medio privilegiado para poner a disposición de la población general los datos, la información y el conocimiento espacial del que disponen.

Teniendo en cuenta este contexto, surge en Zaragoza a principios de 2013 la propuesta de configurar una serie de materiales de trabajo que faciliten a la ciudadanía profundizar en el conocimiento de su propia ciudad para fomentar la interacción ciudadano-información geográfica y favorecer el seguimiento de las actuaciones públicas que afectan a su entorno más cercano (SEBASTIÁN et al, 2014: 857). Con este horizonte, el Centro Ambiental del Ebro (CAE) propone al Grupo de Estudios en Ordenación del Territorio (GEOT) de la Universidad de Zaragoza abordar este proyecto en forma de exposición. El origen de esta iniciativa se remonta a 2001 gracias al impulso de la Agencia de Medio Ambiente y Sostenibilidad del Ayuntamiento de Zaragoza. Con el apoyo de la misma, de otros servicios del Ayuntamiento y de la Asociación para el Desarrollo Estratégico de Zaragoza y su Área de Influencia se han realizado otros trabajos como como «Zaragoza, manzana a manzana» (EBROPOLIS, 2012) o «Valoración de la proximidad de las zonas verdes, equipamientos y servicios en la ciudad de Zaragoza» (Ayuntamiento de Zaragoza, 2013).

\section{OBJETIVOS}

La exposición será denominada «Zaragoza Mapa a Mapa» y se enmarca en la línea de la Neogeografía reconociendo que se ha democratizado el uso y creación de la información geográfica (HAKLAY, 2013: 3).

Este proyecto aborda el objetivo general de involucrar a la sociedad en el conocimiento y valoración de su espacio a través de los siguientes objetivos específicos:

(i) Poner información geográfica al alcance de los ciudadanos fomentando una lectura e interpretación eficiente de la misma. 
(ii) Acercar a los ciudadanos los resultados de la investigación temática llevada a cabo por la Universidad de Zaragoza en relación con la demografía a microescala y la accesibilidad a servicios y equipamientos entendiendo que son determinantes para garantizar la equidad en condiciones de vida en los distintos espacios de la ciudad.

(iii) Recopilar información espacial a través de las redes sociales que los visitantes generen a partir de los materiales expuestos, creando nuevas capas de información que ayuden, maticen y enriquezcan la toma de decisión municipal, y los canales de gobernanza y participación ciudadana en relación al trasporte intraurbano.

\section{METODOLOGÍA}

El marco metodológico se aborda desde el punto de vista del Plan de exposición (Exhibition brief) que describe los diferentes parámetros de la exposición (ALONSO et al, 2010: 31): (i) título, (ii) naturaleza de la exposición, (iii) política y contexto, (iv) material expositivo, (v) espacio de exposición, (vi) visitantes.

\section{III.1. Título y fecha de referencia}

El título completo de la exposición es «Zaragoza mapa a mapa: los servicios a tu lado», con el que se quiere transmitir al gran público una idea principal: la accesibilidad a los servicios y equipamientos públicos y privados son en gran parte la clave de unas condiciones de vida óptimas en la ciudad. En el momento de comenzar el proyecto se contaba con toda la información actualizada a 2012, coincidiendo con los últimos datos padronales disponibles de manera completa.

\section{III.2. Naturaleza de la exposición}

Atendiendo a los diferentes criterios que permiten clasificar una exposición (ALONSO et al, 2010: 2) puede caracterizarse de la siguiente forma: (i) Tener una función documental: puesto que está principalmente ligada al valor informativo y científico de los objetos; (ii) la disposición intencional del mensaje es contextualizada: enfocada en centrar el mensaje a través de una interrelación de valores, (iii) por el tiempo expositivo es temporal y portátil: tuvo una duración limitada, además por su diseño integrado y facilidad de instalación y transporte se pudo instalar en otros espacios diferentes al original, (iv) por la naturaleza del material expuesto es mixta: puesto que incluye material analógico y digital, (v) por la extensión del contenido es polivalente: permite diversos niveles de lectura según diferente mentalidad, nivel de formación y edad, 
(vi) desde el punto de vista del público es además interactiva, puesto que algunos paneles se ven modificados por los visitantes y participativa ya que busca involucrar al visitante.

\section{III.3. Política y contexto}

El proyecto inicial se diseña para exponer en el Centro Ambiental del Ebro, cuyo objetivo es la revitalización de la ciudad y el fomento de actividades saludables, recreativas y educativas que propicien el disfrute de los espacios urbanos y su entorno natural y rural (Centro Ambiental del Ebro, 2015). En su emplazamiento original la exposición se pudo visitar durante tres meses (octubre-diciembre 2013). A lo largo de este periodo se programaron actividades que permitieran que los visitantes interactuaran con la exposición generando nueva información espacial a través de redes sociales y material interactivo.

Además el material ha sido expuesto en otros emplazamientos en el plazo de un año desde que finalizó la exposición principal para garantizar la vigencia temporal de la información. El listado de eventos y espacios es el siguiente: (i) XVI Congreso Nacional de Tecnologías de la Información Geográfica (Alicante, 25-27 junio 2014), (ii) en el Ayuntamiento de Utebo (Mayo-Junio 2014), (iii) Noche de los Investigadores, Universidad de Zaragoza (25 septiembre 2014) y (iv) en la Conferencia ESRI (22-23 octubre 2014).

\section{III.4. Material expositivo}

Este proyecto se basa en el acercamiento de información espacial a usuarios no expertos utilizando la cartografía temática analógica y digital como herramienta de comunicación para conseguir que sean capaces de extraer conclusiones en relación a su entorno. Para esto es necesario preparar el material teniendo en cuenta (i) la temática, (ii) el nivel de complejidad, (iii) el formato y (iv) el diseño cartográfico.

Los temas que se han seleccionado para su desarrollo cartográfico en paneles estructuran el hilo argumental de la exposición y son los siguientes: (i) Desarrollo y cambio de la ciudad, (ii) Espacios verdes, (iii) Equipamientos y servicios de transporte, (iv) Equipamientos culturales y deportivos y por último (v) Valoración de la ciudad.

En referencia al nivel de complejidad, se comienza con cartografía muy sencilla apoyada en imágenes aéreas y con un grado de agregación por juntas de distrito. Progresivamente se incluyen más niveles de información temática y un mayor grado de detalle llegando a la información desagregada por manzanas urbanísticas. De este modo se entrena al visitante en mejorar su proceso de lectura de mapas, consiguiendo que incremente su nivel de formación al 
respecto para abordar con mayor garantía la interpretación de los mapas que muestran más información y presentan un sistema de codificación cartográfica de mayor nivel.

El material utilizado en la exposición se compone de 21 paneles de diferentes medidas que van desde 70 x 50 centímetros hasta el de mayor tamaño que alcanza los 2,80 x 2,10 metros. Además se incorpora un panel interactivo en el que el usuario puede proponer nueva información espacial y se elabora un vídeo que incorpora información de apoyo y toda la cartografía temática que se proyecta en televisiones de 42 pulgadas.

El diseño cartográfico es diferente dependiendo del grado de complejidad de cada uno de los mapas, que se han planteado con base en la clasificación de trayectorias cartográficas (ZúÑIGA, 2009: 276) que permite sistematizar la descripción de cada mapa. Para los más sencillos se opta por información espacial apoyada en imágenes aéreas, delimitación lineal del espacio urbano y utilización de gráficos de barras o sectores. Un segundo nivel introduce información superficial de localización de espacios verdes y de accesibilidad a través de análisis de áreas de influencia consecutivas. Posteriormente se incorpora información puntual que presenta una densidad mayor de información y que se gradúa de manera cualitativa indicando el tipo de equipamiento que representa. Finalmente el mapa de mayor complejidad presenta tres variables: una en superficies graduadas por la variable visual valor mediante una leyenda secuencial y otras dos graduando elementos puntuales a través de símbolos proporcionales volumétricos y combinación de color y valor mediante una leyenda divergente (ZÚÑIGA et al, 2012: 4). Se realiza un análisis más detallado en el apartado correspondiente a resultados.

\section{III.5. Espacio y circulación}

El espacio es, junto con la iluminación, el elemento más determinante para la organización del itinerario expositivo. De la ubicación de los objetos, de la relación que guardan entre sí y con la realidad espacial, depende en gran medida la percepción del material por parte del visitante (ALONSO et al, 2010: 8). La buena organización del espacio en el recorrido global de la exposición, implica no solamente la facilidad y claridad de la circulación para el público, sino también su ritmo (BELCHER, 1991), que consiste en ofrecer al visitante una variedad de experiencia según avanza a través de un espacio determinado. Delimitar el ritmo expositivo en este caso, resulta fundamental, ya que el visitante debe familiarizarse con la información cartográfica más sencilla (imagen aérea y cartografía temática univariable), para poder analizar e interpretar la información específica de su calidad de vida a través de cartografía multivariable compleja. 
La organización del material (FIGURA 1) se realiza de acuerdo a una circulación en peine (LEHMBRUK, 1974: 225), favoreciendo la concentración del material por temáticas (Desarrollo y cambio de la ciudad, espacios verdes, equipamientos y servicios de transporte, equipamientos culturales y deportivos, valoración de la ciudad) y por niveles de complejidad (público no experto, público infantil, público experto).

\section{III.6. Visitantes}

El público objetivo es un usuario no experto en información espacial. Se trata de acercarlo a la misma graduando la complejidad de los mapas y ofreciéndole la cartografía como una herramienta que le permita valorar tanto las condiciones de vida que caracterizan su entorno como las actuaciones públicas que se realizan para mejorarlas. Para facilitar la visita y ampliar el rango de usuarios se propone un espacio para público infantil muy adaptado a su potencial conocimiento del espacio con un diseño de actividades y juegos que permitiese otros modos de conocimiento.

Como ya se ha mencionado en anteriores apartados esta exposición enlaza con el concepto de Neogeografía en la medida en la que asume que la tecnología y las herramientas geográficas han democratizado el uso de la información espacial, en la actualidad el público no experto utiliza y genera geodatos de manera no-formal para sus actividades personales y sociales (GOODCHILD, 2008: 1) y para los procesos de gobernanza y participación ciudadana. Aunque no existe una única definición de Neogeografía sí que aparecen una serie de conceptos clave que son comunes a todas ellas: Web 2.0, GPS, software libre de creación de mapas, participación pública, VGI, contenido generado por usuarios, redes sociales... (RANA Y JOLIVEAU, 2009: 75) Las cuatro últimas están intrínsecamente enlazadas con el proyecto que se presenta en este artículo gracias a un mapa situado en una pizarra interactiva que permite a los usuarios proponer nuevas líneas de transporte sobre la información demográfica preexistente y compartirlas en Twitter con el resto de usuarios.

\section{RESULTADOS}

La exposición queda conformada por 21 paneles, 15 de los cuales son mapas o imágenes aéreas diseñadas, maquetadas e impresas ex profeso para esta exposición en base a investigaciones socio-demográficas realizadas previamente por el grupo de investigación GEOT. En este apartado de resultados se incluye también la información referida a la generación de nuevo contenido espacial a través de las redes sociales y la repercusión que tuvo en las mismas la exposición. 


\section{IV.1. Cartografía realizada}

Dada la imposibilidad de incluir la colección completa de cartografía, se presenta una muestra de las principales tipologías (FIGURAS 2, 3 y 4) y se procede a caracterizar de manera conjunta los mapas incluidos en cada uno de los grandes grupos funcionales (TABLAS 1 a 6), siendo éstos diferentes de los temáticos utilizados de cara a los visitantes: (i) Crecimiento urbano, (ii) Caracterización demográfica, (iii) Localización de hitos, (iv) Accesibilidad a equipamientos y servicios, (v) Propuesta de recorrido líneas nuevas de transporte público y (vi) Valoración de la accesibilidad. La descripción de la cartografía se realiza a partir de los siguientes parámetros: (i) público objetivo, (ii) número de paneles, (iii) grado de agregación, (iv) nivel de complejidad, (v) formato, (vi) reflexión que se espera del visitante y (vii) diseño cartográfico. Los formatos digitales proyectados a partir de televisión han permitido la realización de zoom y mayor interactuación con los usuarios mientras que los analógicos permiten una reflexión más pausada.

\section{IV.2. Interacción con el visitante a través de redes sociales:}

Tal y como se ha indicado en esta exposición se ha utilizado el potencial de Twitter para interactuar con el usuario no experto. El objetivo era conseguir por un lado la retroalimentación y publicidad respecto a la valoración de la exposición (FIGURA 5) y por otro la generación de nueva información espacial en lo que refiere a propuestas de futuras líneas de tranvía (FIGURA 6).

\section{DISCUSIÓN}

La esencia de la Neogeografía radica en asumir que se ha producido un fenómeno de democratización y apertura de la información geográfica facilitando el acceso, edición y difusión por parte de usuarios no expertos para el uso personal (BUTLER, 2006: 777). No obstante, algunos autores defienden que esta democratización tiene una serie de limitaciones (HAKLAY, 2013: 56). Desde la perspectiva de este trabajo se considera que el acceso a información geolocalizada parece superado, sin embargo uno de estos límites aparece a la hora de utilizar información espacial enriquecida con información temática, en concreto sociodemográfica y de accesibilidad. En este caso el usuario no experto no encuentra la facilidad de uso y sobre todo las herramientas de interpretación necesarias que le permitan razonar más allá de la observación generando un análisis más profundo de la realidad espacial en la que vive.

Bajo estas premisas, se considera que iniciativas como la presentada en este proyecto, dotan al ciudadano del conocimiento suficiente para argumentar de una forma colectiva y reflexionada en los procesos de planificación y gestión urbanas. Esto se pone de manifiesto en el material interactivo existente en la 
exposición en relación con la propuesta de nuevas líneas de tranvía. Los ciudadanos sin información adicional sobre su ciudad (número de habitantes por barrio, accesibilidad desde su barrio a otras alternativas de transporte...) velan por sus intereses y plantean propuestas directas sobre su entorno. Sin embargo, tras realizar la visita a la exposición y comprobar las diferentes variables que intervienen en la ordenación territorial de Zaragoza, apuestan por estrategias más equitativas que abarquen las necesidades de una población más extensa (las rutas se codificaban en este caso, teniendo en cuenta la densidad de población de los barrios y su menor acceso a otras alternativas de transporte), primando estos criterios frente a su intereses particulares (SEBASTIÁN et al, 2014: 864).

Resulta evidente que la «ciencia ciudadana» puede abarcar mucho más que la simple recolección de datos y tiene el potencial de posibilitar la participación de la sociedad con su ciudad o territorio de residencia. Iniciativas de este tipo cubren la exigencia de la ciudadanía, conjugando prácticas de gestión (aumento de las relaciones con los agentes del conocimiento y participación de ciudadanos) en los procesos de toma de decisiones. Desde este equipo de investigación se apuesta por soluciones híbridas (GOODCHILD, 2008: 16) que permiten enriquecer la información espacial a partir no solo de la visualización y recolección de datos, sino de herramientas de trabajo colaborativas que ayuden, entre otros aspectos, a jerarquizar y crear opinión sobre las actuaciones, a favorecer la gestión eficiente, o a realizar un seguimiento de las actuaciones en el territorio.

Las perspectivas a futuro tienen un amplio recorrido para el material preparado en Zaragoza Mapa a Mapa. El primer paso consiste en mantener actualizados los mapas para lo que es imprescindible conectar la cartografía directamente con las bases espaciales que se representan y vincular a la ciudadanía en esta actualización de la información (Ayuntamiento de Zaragoza, 2015). Además se están desarrollando propuestas vinculadas a plataformas web (FIGURA X) que permiten una mayor interacción y flexibilidad de utilización al usuario desde diferentes dispositivos (ordenador, tablet, móvil). Se realiza a través de tecnología de visualización de información espacial basada en el framework de código abierto OpenLayers, y complementada con la librería JQuery (biblioteca de JavaScript), que permite simplificar la manera de interactuar con los documentos HTML, manipular el árbol DOM (Document Object Model), manejar eventos, desarrollar animaciones y agregar interacción con la técnica AJAX (Asynchronous JavaScript And XML) a páginas web para la creación de interfaces gráficos (ZÚÑIGA et al, 2015: 5).

Por otro lado se apuesta por poner en valor la información geográfica colaborativa que deberá tener plataformas que la canalicen, sin saturar, con nuevas 
aplicaciones sino que puedan aprovechar el potencial de las redes sociales. En la actualidad la Sede Electrónica del Ayuntamiento ya ofrece a la ciudadanía un servicio multicanal personalizado de acceso desde todo tipo de dispositivos.

\section{AGRADECIMIENTOS}

Este trabajo ha sido realizado gracias al impulso de la Agencia de Medio Ambiente y Sostenibilidad del Ayuntamiento de Zaragoza y la Asociación para el Desarrollo Estratégico de Zaragoza y su Área de Influencia (Ebrópolis). Queremos destacar el inestimable apoyo del Centro Ambiental del Ebro, quien proporciona el material económico, técnico y expositivo necesario, para poder llevar a cabo la muestra cartográfica que se presenta. Las actualizaciones del mismo se enmarcan en el proyecto «Herramientas cartográficas para una gobernanza inteligente en las ciudades digitales: análisis territorial de las condiciones de vida» (CSO2013- 46863-C3-3-R) del Programa Estatal de Investigación, Desarrollo e Innovación Orientada a los Retos de la Sociedad del Ministerio de Economía y Competitividad de España y en los contratos de investigación con la Universidad de Zaragoza 2013/1126 «Organización de la exposición Zaragoza Mapa a Mapa» y 2014/0466 «Referenciación de la información del padrón municipal por portales y desarrollo de herramientas cartográficas para una gobernanza inteligente en la ciudad de Zaragoza».

\section{BIBLIOGRAFÍA}

Alonso, L. y GarcíA, I. (2010): Diseño de exposiciones. Concepto, instalación y montaje. Madrid, Alianza Forma.

AYUNTAMIENTO DE ZARAGOZA (2013): «Valoración de la proximidad de las zonas verdes, equipamientos y servicios en la ciudad de Zaragoza».

https://www.zaragoza.es/ciudad/medioambiente/agenda21/observatorio/estudiosindicadores.htm consultado el 08/02/2015.

AYUNTAMIENTO DE ZARAGOZA (2015): «IDEZar». http://www.zaragoza.es/ciudad/idezar/ consultado el 02/02/2015.

BELCHER, M. (1991): Exhibitions in museums. Leicester, Leicester University Press.

ButLeR, D. (2006): «The Web-Wide World». Nature, $\mathrm{n}^{\circ}$ 439, 776-778.

CALVO, J.L.; PUEYO; A. y ZÚÑIGA, M. (2011): «La ciudad de Zaragoza en un escenario de crisis diagnóstico y propuestas territoriales para nuevos paradigmas urbanos». Geographicalia, no 59-60, 47-60.

Centro AmbientAl Del EBro (2015): «Presentación y objetivos». http://www.zaragoza.es/ciudad/centroambientalebro/presentacion.htm consultado el 08/02/2015.

Cohn, J. (2008): «Citizen Science: Can Volunteers Do Real Research?» BioScience, $\mathrm{n}^{\circ} 58$ (3), 192-197. 
Dickinson, J.; ZuckerberG, B.; y BonTeR, D. (2010): «Citizen Science as an Ecological Research Tool: Challenges and Benefits». Annual Review of Ecology, Evolution, and Systematics, $\mathrm{n}^{\circ}$ 41, 149-172.

EBROPOLIS (2015): «Zaragoza manzana a manzana» http://observatoriourbano.ebropolis.es/web/observatoriourbano/manzanaamanza na.asp consultado el 07/01/2015.

ELWOOD, S. (2008): «Volunteered geographic information: key questions, concepts and methods to guide emerging research and practice». GeoJournal, n ${ }^{\circ}$ 72, 133135.

GoODCHILD, M. (2008): Assertion and authority: the science of user-generated geographic content. Berkeley, University of California.

HAKLAY, M. (2013): «Neogeography and the delusion of democratization». Environment and Planning A, $\mathrm{n}^{\circ}$ 45(1), 55-69.

LEHMBRUCK, M. (1974): «Functions. Space and circulation». MUSEUM magazine, $\mathrm{n}^{\circ}$ 3/4, 221-236.

RANA, S. y JoliVeaU, T. (2009): «Neogeography: an extension of mainstream geography for everyone made by everyone?» Journal of Location Based Services, $n^{0} 3(2), 75-81$.

Sebastián, M.; ZÚÑiga, M.; Pueyo, A.; Postigo, R.; Salinas, C.; ArranZ, A. y LÓPEZ, C. (2014): «Zaragoza Mapa a Mapa: una herramienta para el ciudadano». Ponencia en XVI Congreso Nacional de Tecnologías de la Información Geográfica, (Alicante, 25-27 de junio de 2014). Alicante, Universidad de Alicante, 856864.

ZÚÑIGA, M. (2009): Propuesta cartográfica para la representación y análisis de las variables de población mediante sistemas de información geográfica e infografía: el caso español. Zaragoza, Prensas Universitarias Universidad de Zaragoza.

ZúNiga, M.; PueYO, A., y CALVO, J. (2012): «The Spanish population during the twentieth century and beyond». Journal of Maps, $\mathrm{n}^{\circ}$ 8, 386-391.

ZÚÑIgA, M.; SEbastián, M.; PÉRez, MJ.; Fernández, MJ; Alonso, M.P. y Pueyo, A. (2015): «Visualización y análisis de información sociodemográfica a través de los servicios IDEZar del Ayuntamiento de Zaragoza». Ponencia en XXIV Congreso de la Asociación de Geógrafos Españoles (Zaragoza, 28 al 30 de octubre de 2015). Zaragoza. 
Figura 1. Espacio y circulación en la exposición.

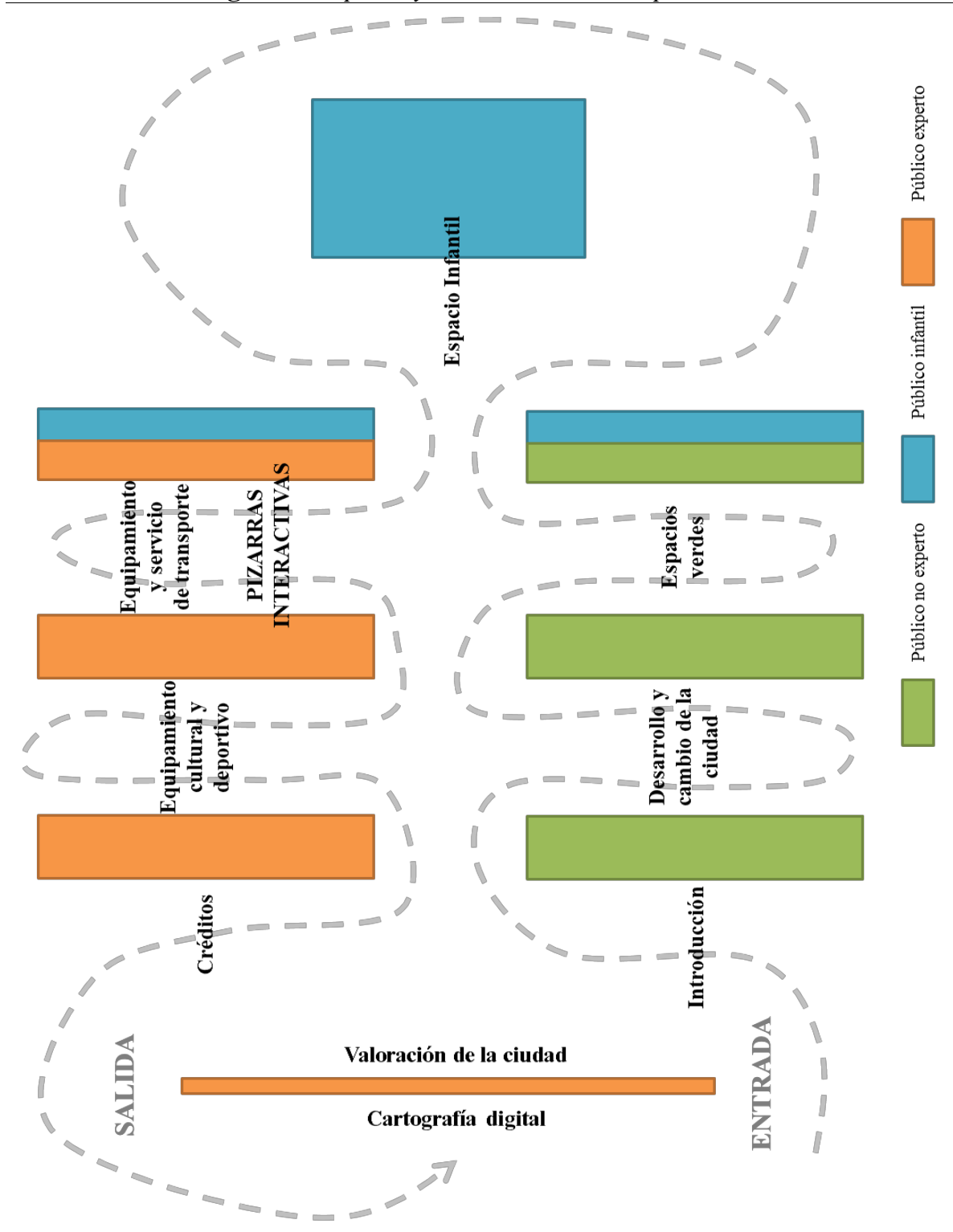

FuENTE.: Grupo de Estudios en Ordenación del Territorio, Universidad de Zaragoza, 2015 
Figura 2. Evolución del continuo urbano en Zaragoza.

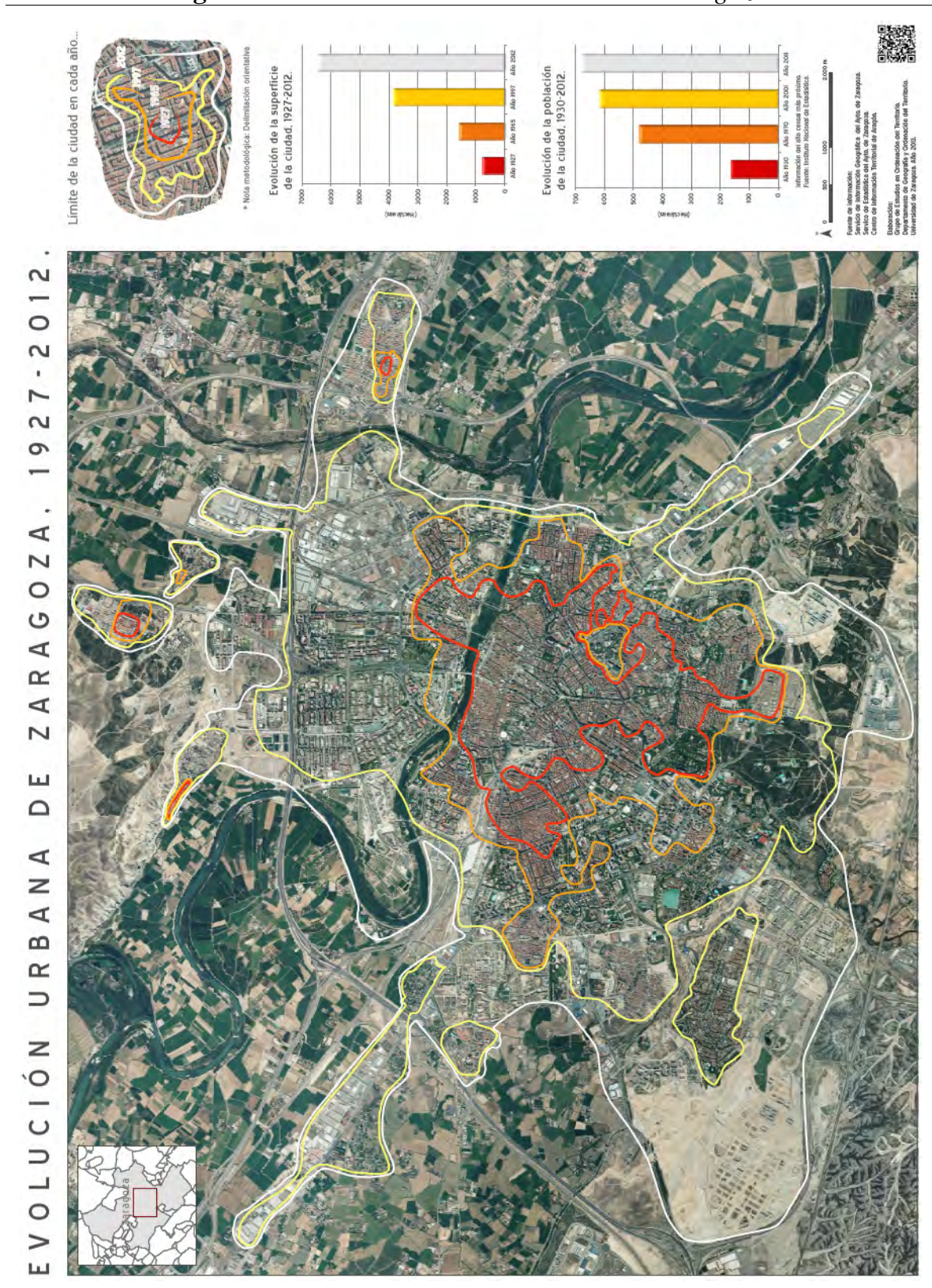

FUENTE.: Grupo de Estudios en Ordenación del Territorio, Universidad de Zaragoza, 2013 
Figura 3. Disponibilidad de museos, bibliotecas, cines y teatros.
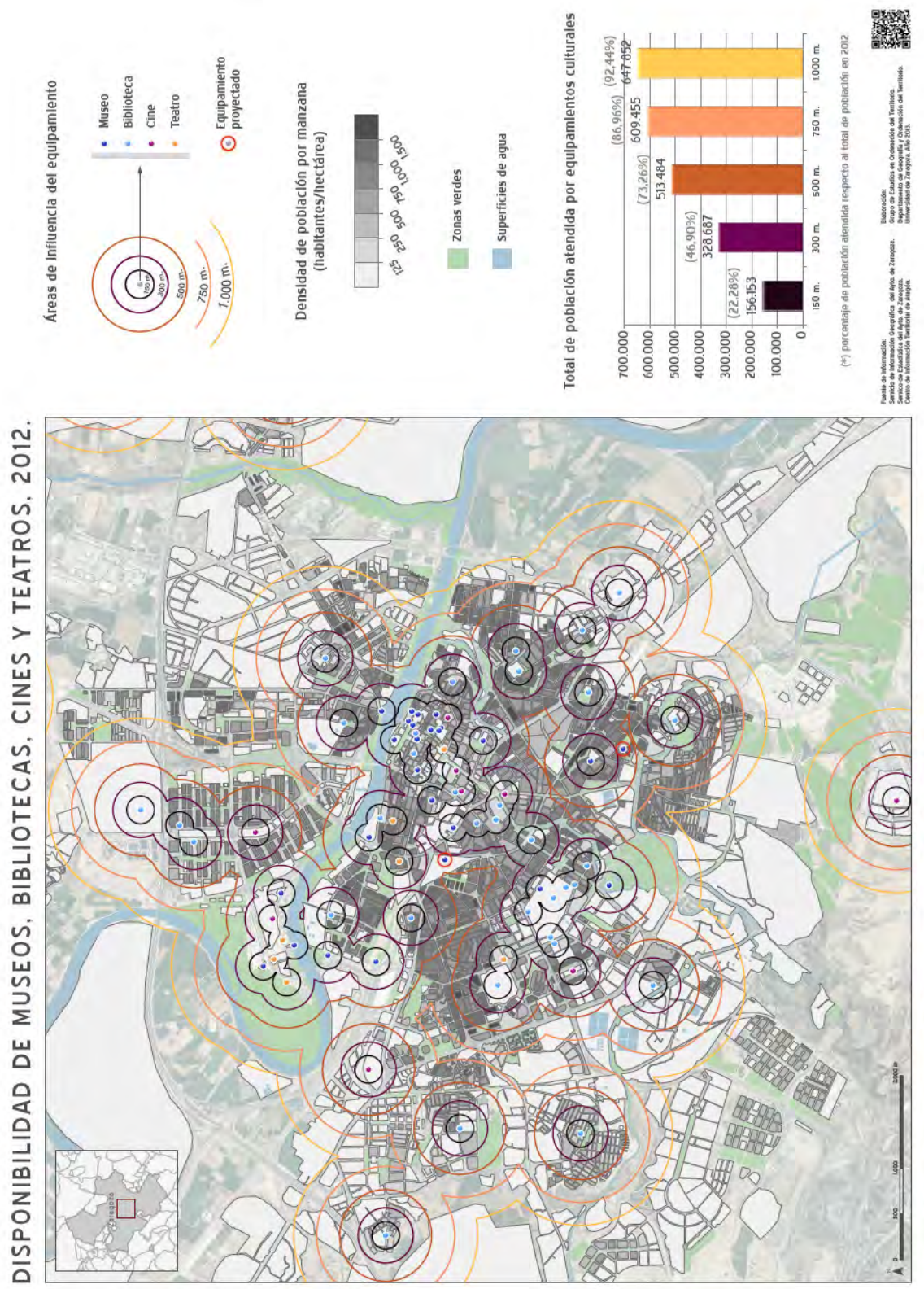

FuENTE.: Grupo de Estudios en Ordenación del Territorio, Universidad de Zaragoza, 2013 
Figura 4. Valoración de la accesibilidad a los equipamientos básicos en la ciudad de Zaragoza.

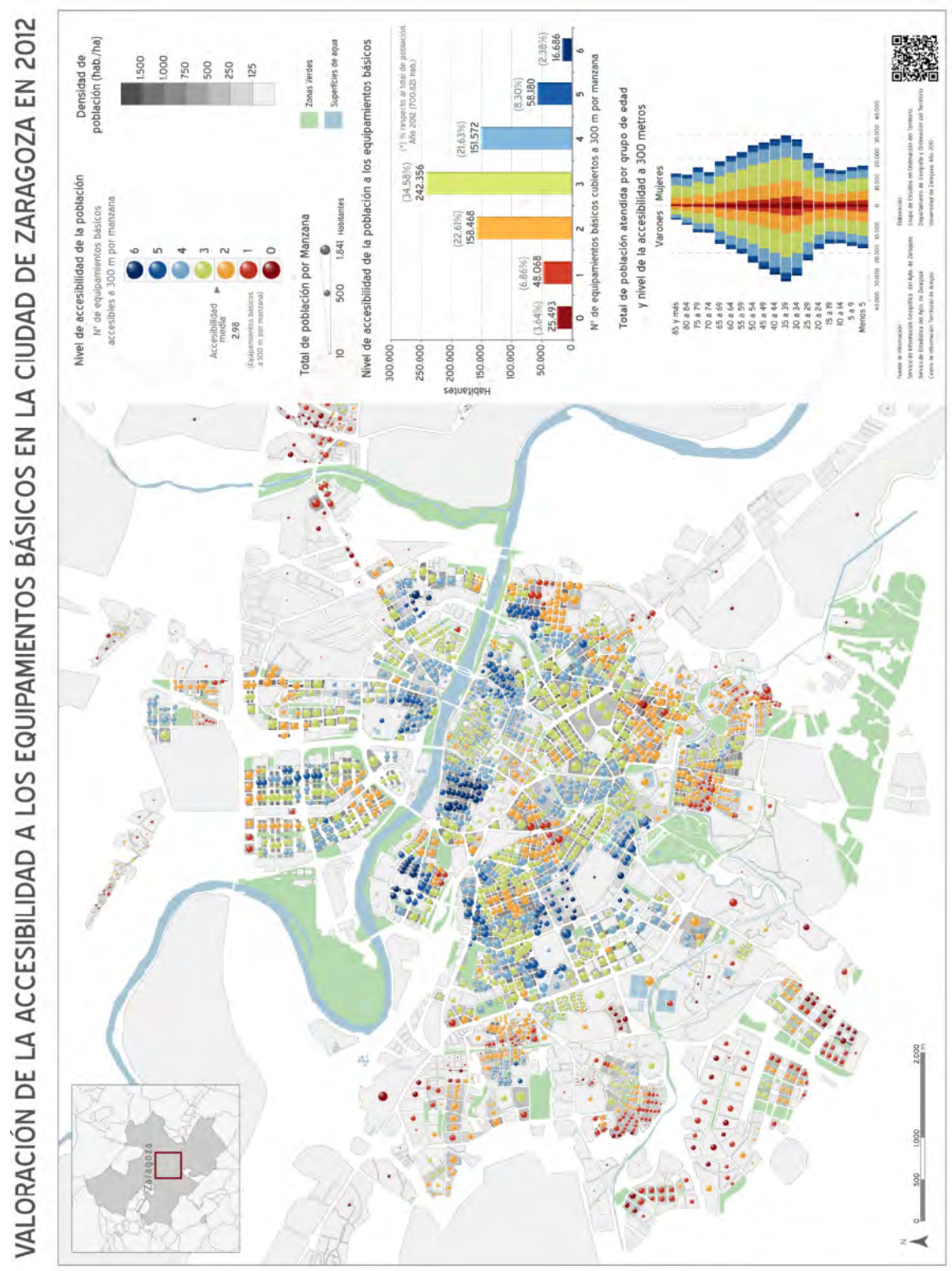

FUENTE.: Grupo de Estudios en Ordenación del Territorio, Universidad de Zaragoza, 2014 
Tabla 1. Valoración de la accesibilidad a los equipamientos básicos en la ciudad de Zaragoza.

Público objetivo, No experto; $N^{o}$ paneles, 3; Grado de agregación, Ciudad completa; Nivel de complejidad, Bajo; Formato, Analógico y digital; Reflexión del visitante, ¿Qué había antes donde vivo ahora?

Diseño cartográfico: dos de los paneles muestran por un lado la comparación de espacios en imagen aérea de 1927 y 2012. El tercero muestra la delimitación del continuo urbano, información cualitativa mostrada a través de implantación lineal graduada por color

\section{Limite de la ciudad en cada año...}
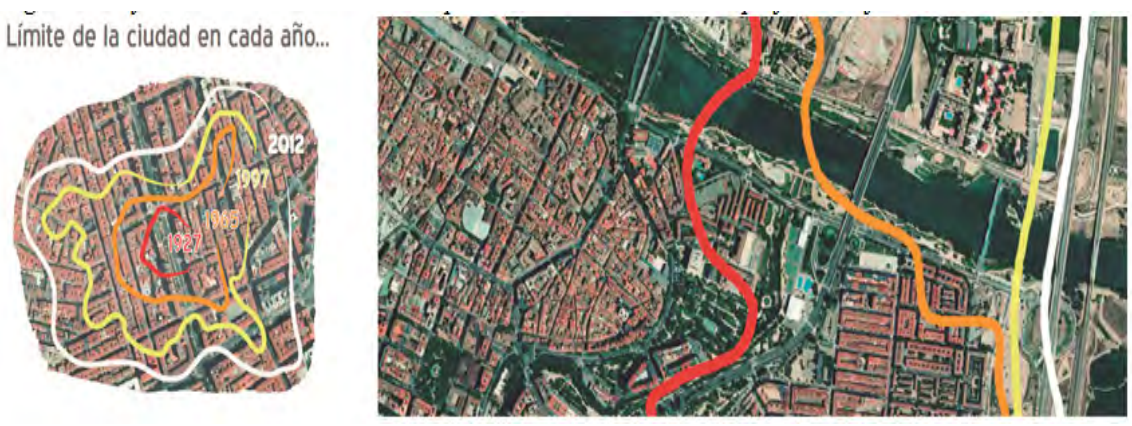

FuENTE.: Grupo de Estudios en Ordenación del Territorio, Universidad de Zaragoza, 2015

Tabla 2. Caracterización demográfica.

Público objetivo, No experto; $N^{\circ}$ paneles, 2; Grado de agregación, Juntas de distrito; Nivel de complejidad, Bajo; Formato, Analógico; Reflexión del visitante, ¿Cómo es la gente de mi barrio? ¿Cuántas personas viven en él?

Diseño cartográfico: son dos paneles con información demográfica de naturaleza cuantitativa que se representan mediante implantación puntual, bien ligada a una proporcionalidad del tamaño o a gráficos de sectores
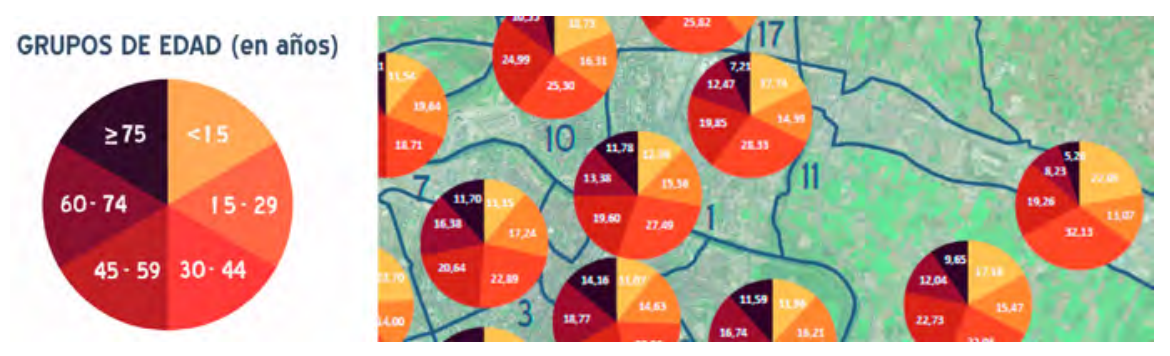

FUENTE.: Grupo de Estudios en Ordenación del Territorio, Universidad de Zaragoza, 2015 
Tabla 3. Localización de hitos.

Público objetivo, Infantil; $N^{\circ}$ paneles, 1; Grado de agregación, Ciudad completa; Nivel de complejidad, Bajo; Formato, Analógico; Reflexión del visitante, ¿Dónde están mi casa y mi colegio? ¿Dónde estoy ahora? ¿Cómo llego de un sitio a otro?

Diseño cartográfico: es una impresión de la imagen aérea de la ciudad sobre un panel de 2x2 m, dispone de elementos que permiten interactuar (fichas de hitos de la ciudad, coches de juego...)
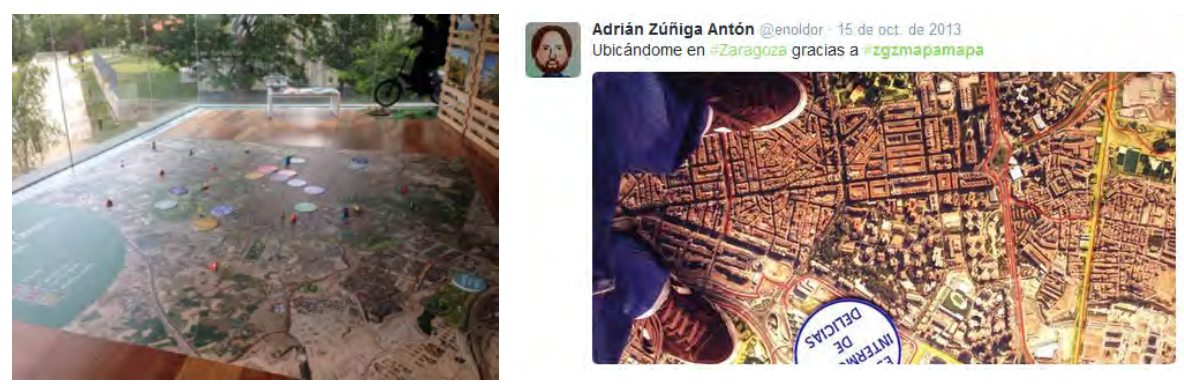

FuENTE.: Grupo de Estudios en Ordenación del Territorio, Universidad de Zaragoza, 2015. Zúñiga, A. (enoldor). «Ubicándome en \#Zaragoza gracias a \#zgzmapamapa http://t.co/uo8OD2bzGv». 15 Oct 2013, 10:41 UTC. Tweet

Tabla 4. Accesibilidad a equipamientos y servicios.

Público objetivo, Experto; $N^{o}$ paneles, 6; Grado de agregación, Manzana; Nivel de complejidad, Medio-Alto; Formato, Analógico y digital; Reflexión del visitante, ¿Cuál es el parque más cercano a mi casa? ¿Cuánta gente utiliza la misma parada de tranvía que yo? ¿Dónde ha habido una mayor inversión por parte del ayuntamiento?

Diseño cartográfico:son composiciones cartográficas con tres niveles de lectura: el primero actúa como fondo utilizando implantación superficial graduada por valor en una leyenda secuencial, el segundo localiza los equipamientos mediantes implantación superficial o puntual y representa las categorías mediante el color por último mediante implantación lineal graduada por valor se visualizan las áreas de influencia

Áreas de influencia del equipamiento
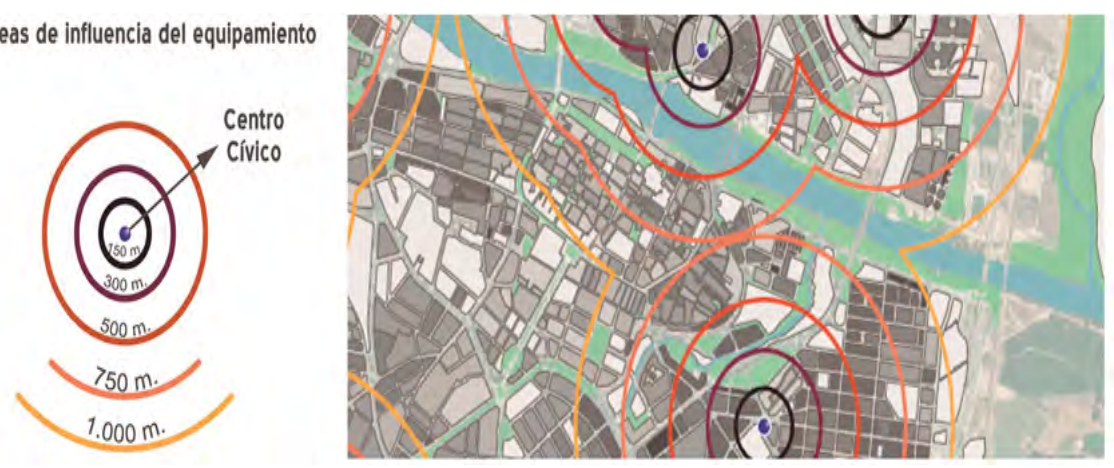

FUENTE.: Grupo de Estudios en Ordenación del Territorio, Universidad de Zaragoza, 2015 
Tabla 5. Propuesta de recorrido líneas nuevas de transporte público.

Público objetivo, Experto; $N^{o}$ paneles, 1; Grado de agregación, Manzana; Nivel de complejidad, Medio-Alto; Formato, Analógico; Reflexión del visitante, Si tuviera que proponer una nueva línea de transporte público ¿Por dónde pasaría?

Diseño cartográfico:se incorpora un fondo de contextualización temática utilizando información cuantitativa sobre implantación superficial graduada por valor y una serie de elementos de apoyo mediante líneas (trayectos preexistentes) y puntos (intercambiadores, parking disuasorios). Se espera que el visitante formule nuevas alternativas a la segunda línea del tranvía y envíe su propuesta mediante Twitter a \#zgzmapamapa

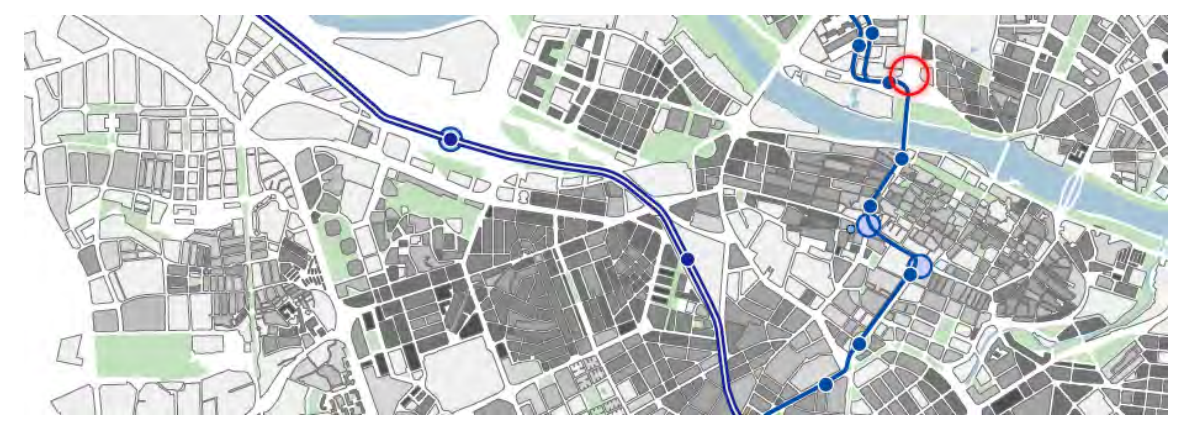

FuENTE.: Grupo de Estudios en Ordenación del Territorio, Universidad de Zaragoza, 2015

Tabla 6. Valoración de la accesibilidad.

Público objetivo, Experto; $N^{\circ}$ paneles, 1; Grado de agregación, Manzana; Nivel de complejidad,Alto; Formato, Analógico y digital; Reflexión del visitante, Si me fuera a cambiar de casa ¿En qué zona buscaría? ¿Dónde ha habido una mayor inversión por parte del ayuntamiento?

Diseño cartográfico:Es una composición cartográfica con dos niveles: Por un lado el mismo fondo de contextualización demográfica que aparece en los mapas anteriores y por otro un nivel más complejo que superpone dos variables cuantitativas sobre implantación puntual. La primera mediante dimensionamiento volumétrico del tamaño y la segunda mediante combinación de color y valor en una leyenda divergente

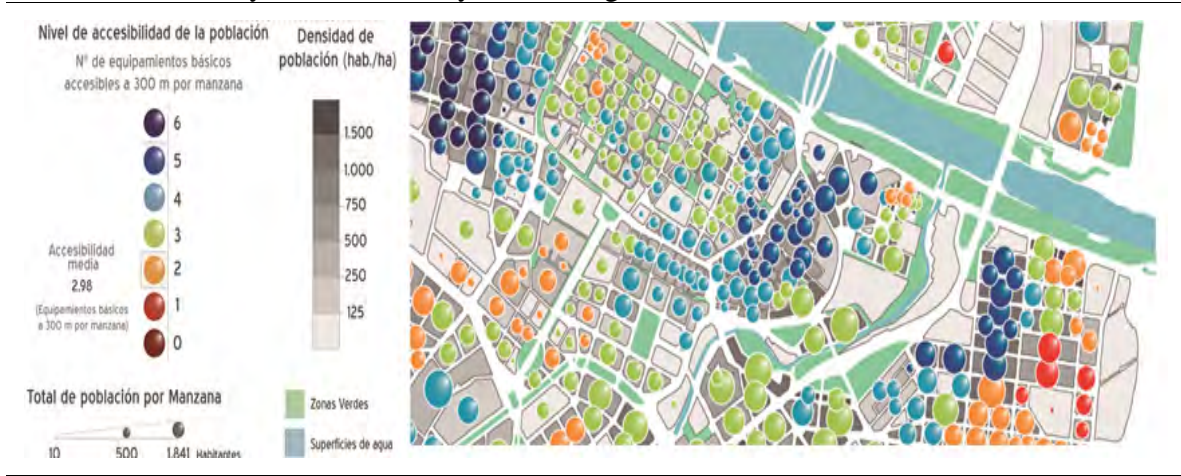

FUENTE.: Grupo de Estudios en Ordenación del Territorio, Universidad de Zaragoza, 2015 
Figura 5. Tweets referidos a la evaluación externa y publicidad de la exposición

EcoTecnix @EcoTecnix - 10 de nov. de 2013

Hoy a tocado visita a la exposición : Zgzmapamapa, muy recomendable el estudio de @GEOT_zgz para conocer mejor el desarrollo de la ciudad.

samuel barrao@SamuelBarrao-4 de dic. de 2013

Vaya mapa chulo@GEOT_zgz@Ireneejea16 @ppipermin @anaagudogil3

+zgzmapamapa

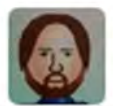

Adrián Zúñiga Antón @enoldor - 15 de oct. de 2013

Para conocer más la ciudad vente a =zgzmapamapa

ㄴา 1

FUENTE: EcoTecnix (EcoTecnix). «Hoy a tocado visita a la exposición \#Zgzmapamapa, muy recomendable el estudio de @GEOT_zgz para conocer mejor el desarrollo de la ciudad». 10 Nov 2013, 13:45 UTC. Tweet / Barrao, S. (SamuelBarrao). «Vaya mapa chulo@GEOT_zgz \#zgzmapamapa http://t.co/yDrWNwoe0s».04 Dec 2013, 17:54 UTC. Tweet / Zúñiga, A. (enoldor). «Para conocer más la ciudad vente a \#zgzmapamapa». 15 Oct 2013, 10:39 UTC. Tweet

Figura 6. Tweets referidos a la evaluación externa y publicidad de la exposición
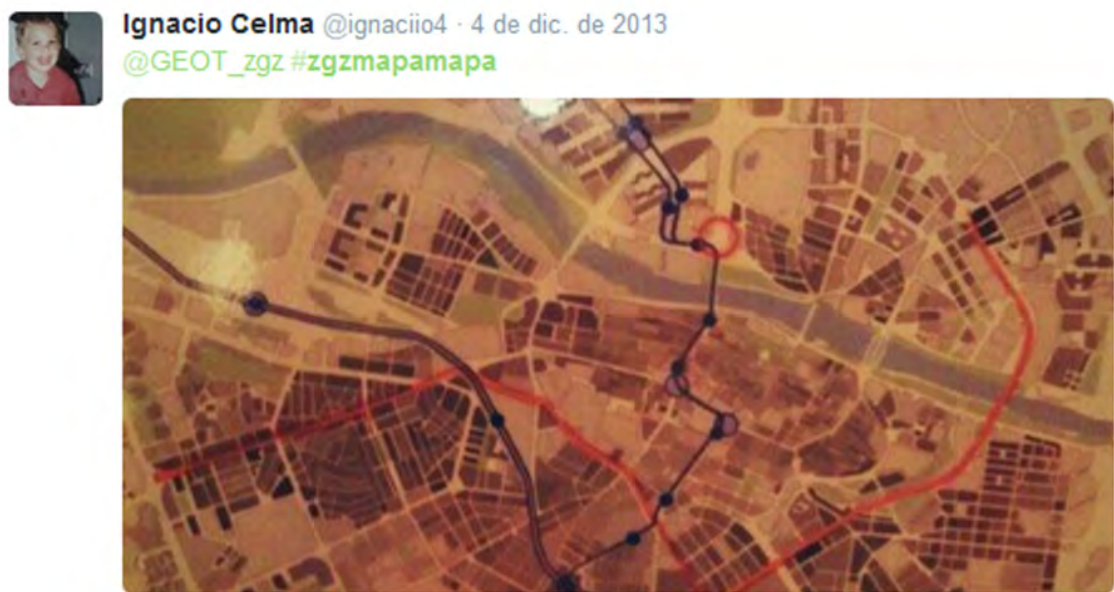

FUENTE: Celma, I. (ignaciio4). «@GEOT_zgz \#zgzmapamapa http://t.co/keWpFL43cP». 04 Dec 2013, 18:10 UTC. Tweet 
Figura 7. Visor de información sociodemográfica basado en la temática de exposición Zaragoza Mapa a Mapa
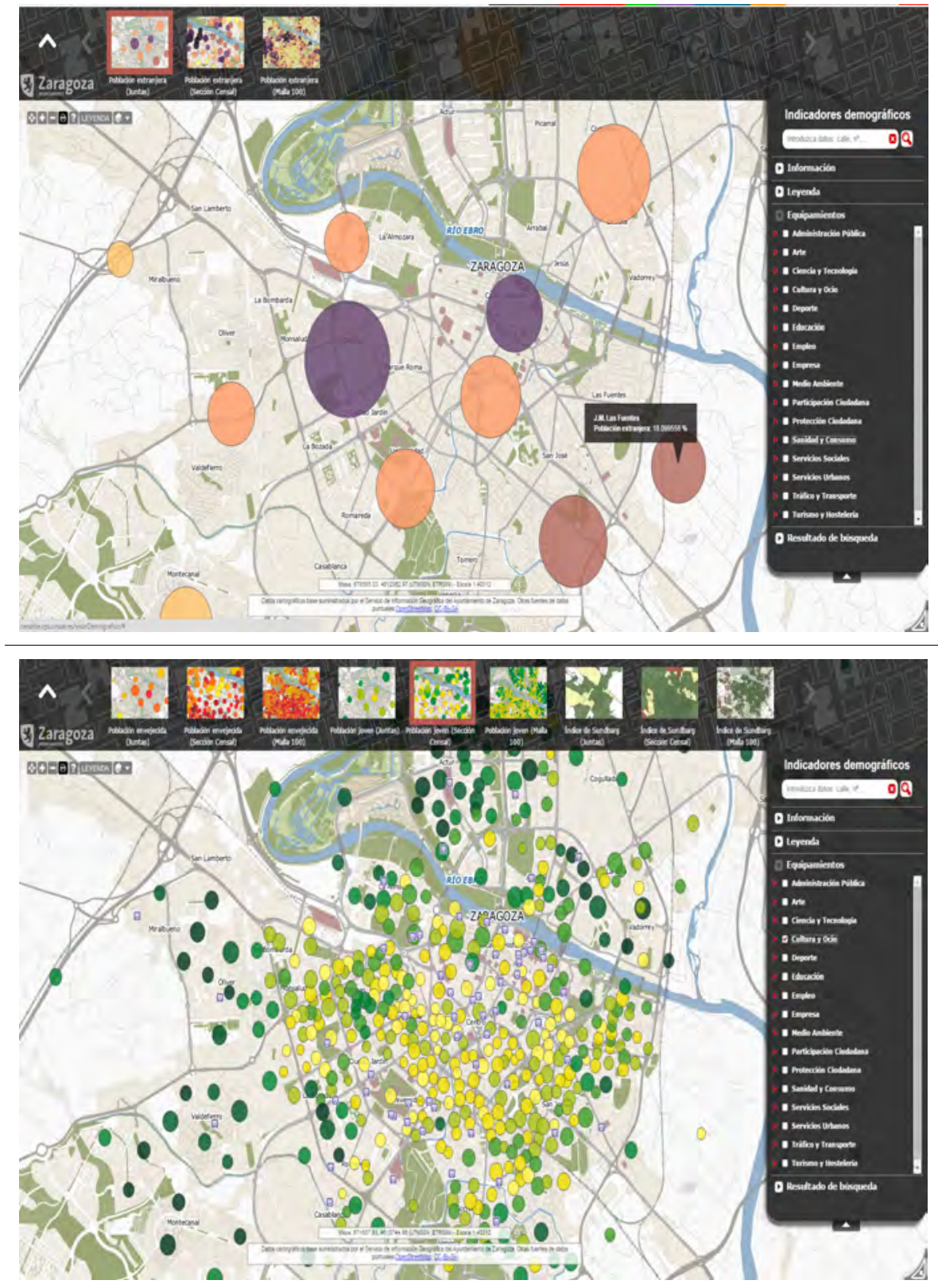

FUENTE: Elaboración propia, 2015 